\title{
The Technical Solutions of Intelligent Venue and the Composition of Its Business Module
}

\author{
Shuyi $\mathrm{Wu}^{1}$, Ye Jin ${ }^{2}$, Jiankang Zhang ${ }^{3 *}$ \\ ${ }^{1,3}$ School of International Economy\&Tourism Management,Zhejiang International \\ StudiesUniversity, Hangzhou,Zhejiang 310023, China \\ ${ }^{2}$ School of English Studies,Zhejiang International Studies University,Hangzhou,Zhejiang 310023, China \\ *Corresponding author.Email: zhangjk@zisu.edu.cn
}

\begin{abstract}
Speech recognition solution, database solution and AI application solution are three technical solutions of intelligent venue.The business module of speech recognition solution includes intelligent speech interaction module and AIUI module.The business module of the database solution includes data backup module,log data module,data collection and annotation module, and information flow module. The business module of AI application solution includes location based service module, face recognition module and data collection and marking module.Exhibition enterprises should further explore the technical solutions of intelligent venue and innovate business module design to meet the needs of conference intelligence operation.
\end{abstract}

Keywords: intelligent venue, speech recognition, business module

\section{INTRODUCTION}

The development of intelligent technology has been having a subversive impact on all walks of life, and the traditional conference forms in many cities are also changing.Intelligent conference has been booming.Based on the intelligent technological means of information and intelligence,the venue provider has created a new form of operation and management of the conference venue,realizing the integrative development of site selection,conference implementation and service delivery. The core technology type of intelligent venue mainly includes speech recognition, database and AI application, and the design of business modules of these three technology types plays a key role in the successful operation of intelligent venue. With the increasingly mature speech recognition technology,intelligent venue can deal with speech processing with ease; provide a mass of data and standardized interface to adapt to more meeting scenes; AI technology enables human intelligence through computers,and increasingly superior system ensures its professionalism and reliability.

\section{SPEECH RECOGNITION SOLUTION}

Speech recognition technology refers to the feature analysis of one or more venue speech signals to realize the recognition and calculation analysis of the venue voice.Speech recognition applies artificial intelligence (AI) to speech recognition technology,providing powerful technical support in the process of model building,speech signal feature extraction and analytic processing.Speech recognition technology uses a combination of the cloud and local speech recognition algorithm,taking advantage of the large database to achieve the goal of recognizing a lot of voice of venue,with the help of a learning calculation method which is similar to artificial neural network,computing distributed parallel depth information to improve accuracy of speech recognition, which is the important way to realize intelligent venue.The business module of speech recognition solution includes intelligent speech interaction module and AIUI module[1].

\subsection{Intelligent SpeechInteractionModule}

Intelligent speech interaction module can be applied in a variety of realistic scenarios of exhibition industry by enabling artificial intelligence technology (AIT) to recognize speech,synthesize speech and "understand" natural language,giving the event an intelligent humancomputer interaction experience about"listening,speaking and understanding".

Natural language processing (NLP) application programming interface (API) can help with venue setup,data search,content recommendation,real-time situation monitoring and analysis,text structuring,etc.,as well as smart products such as intelligent conversational robots.It can recognize real-time radio in one second, and the speed of transferring is extremely fast,which can achieve the effect of "writing while speaking"[2].The built-in intelligent segmentation system will automatically make pauses in sentences after the text is transferred in large sections,forming text paragraphs that are conducive to readers' reading and can be useddirectly. The solution can also be used for real-time live video subtitles, real-time meeting notes,intelligent voice assistant and other scenes,meanwhile it can customize personalized solutions through cooperation. 
Based on intelligent rules, intelligent dialogue can recognize and analyze the contents of the dialogue from a large number of dialogue recordings or dialogue texts,improve the service quality of the venue,facilitate all parties, and optimize service strategies. On the premise of users' participation in the design,constantly perfecting the interactive design process of input - processing - output of speech interaction.Underthe real-time live subtitles and the scene of monitoring of live presentation and live,speech interaction system can transfer the audio in the video into subtitles in real time.In the live scene,the content can be further monitored.

\subsection{AIUI Module}

AIUI is a human-computer interaction system which takes speech as the core designed to use semi-supervised optimization of applications and devices and provide optimization suggestions to developers by analyzing products' logs.At the same time, through the full link and scene dynamic vocabulary, it can achieve the effect of rapidly possessing the capacity of listening,speaking,understanding and thinking at all kinds of venue scene[3]."Software + service","software + application" mode makes the system quickly become a high-quality configuration scheme of intelligent venue.In this module,the wake-on rate of wake-on-voice technology is above 95\%, and meanwhile with low configuration requirements and lowpower consumption,the system has conducted intelligent evaluation on the quality of the wake-on words.

Natural language understanding technology helps developers to customize speech interaction skills by $120+$ depth customization techniques and perfect visual editing page provided by the official,and relying on the intelligent learning ability of the machine,long-standing massive data and strong echo cancellation ability of the microphoneit is able to identify the complicated semanteme and make an accurate response.

Speech synthesis technology supports both online and offline speech synthesis.It can provide a lot of unique timbre that is close to or even beyond the human voice,supporting Chinese,English,Cantonese and even dialects.The traffic consumption of average word is less than $100 \mathrm{~b}$, and the memory usage is very low.

\section{DATABASE SOLUTION}

Information technology has changed the information processing mode and transmission mode of traditional database.The development of intelligent venue to a great extentbenefits from data exchange.As the data of the core attraction of the venue, the database will provide persistent support and services,so that the venue can obtain the core competitive advantage in the data era.In the future,database technology will be integrated with network communication technology,multimedia technology,artificial intelligence (AI) technology and other technologies to achieve multifield penetration, and make optimization of process, integration of related information and data management of the venue in the whole process of venue operation. The business module of the database solution includes data backup module,log data module,data collection and annotation module,and information flow module.

\subsection{Data Backup Module}

The traditional venue faces a series of problems of the long time and low efficiency of data backup; broadband network's large consumptionof massive data backup; large occupation of storage space of backup copy; low expandable space of room storage system.But data backup module can share backup resources,realize centralized backup management, and the time of data recovery is short.Based on the snapshot technology and mirroring technology, the data backup and transmission between the database and the virtual machine can be realized quickly to improve the working efficiency of intelligent venue[4].Ensuring the operation of the business system as a priority,and implementing staggering transfer of backup data; The optimized data redelete technology not only ensures the backup effect,but also greatly reduces the network consumption,finally achieves the goal of optimizing the venue service.

The user layer,the interface layer,the function layer and the resource layer interflow with each other to effectively deal with the backup data of different business value and generate the optimal project of the venue.At the same time, it supports the remote location of host and backup,supports the multiplex reserve mechanism of two places and three centers,and the cloud storage is no longer capped,so as to better serve the intelligent venue business.

\subsection{Log Data Module}

Log data to some extent reflects the organization's structure,daily work patterns and various abnormal conditions.Making use of $\log$ data to analyze the venue,which is helpful for the organizer to monitor the operational aspect of the venue in real time,find the deficiencies of the venue and adjust the project in time.

Using the search page of log service console,you can quickly retrieve the abnormal event log by using the abnormal keywords.For example,the invocation information of abnormal events can be filtered out completely by searching and tracking.Meanwhile,fuzzy query and range query are supported to facilitate quick positioning and business operation.

The combination of data-centered analysis algorithm and human-centered interactive visualization can play to theanalysis strengths of both algorithm and human at the same time.The visual analysis method can effectively 
customers,with AI big data to analyze the surrounding traffic and hotel accommodation,calculate the best exhibition location through relevant systems and provide targeted location service solutions.

Based on the specific scenes of the logistics industry,we provide customized site selection service solutions whichstart from the actual scenes of investment attraction and distribution planning.Customizing exclusively route strategy for freight cars to avoid traffic restrictions, height and weight limit sections and reduce management costs. Making customized search strategy and address analytic optimization for specific exhibitions[6].

Setting up deep (in-depth) cooperation with CAOCAO,Uber taxietc.those industry leading enterprises, which have a deep understanding of travel demand scenarios and pain points of the audience and exhibitors,can provide more professional LBS capacity support and can meet the complex scenes of multistart,multi end,multiple transportation modes and so on to further optimize exhibition service.

\subsection{Face Recognition Module}

\subsection{Information Flow Solution Module}

File processing links up with object storage,and through simple configuration and authorization, the venue can automatically open a series of functions such as file transcoding,watermarking,snapshotting,auditing,streaming ,etc.,to complete the document processing in a one-stop way.

For all kinds of application scenarios such as news client,Feed flow recommendation, it can provides one-stop cloud service of targeted information,management,storage, distribution and so on for the exhibition.With TPG picture acceleration,the client users can also reduce the cost of network traffic, which is beneficial to make great efforts to publicize for the venue,improve the user experience and increase customer stickiness.

\section{AI APPLICATION SOLUTION}

AI application refers to that the AI system establishes algorithm model in the virtual environment,and work out the best venue plan,pre-exhibition site selection,face recognition report and registration,post-exhibition data collection,etc.through the iterative optimization of AI technology to serve intelligent venue. The business module of AI application solution includes location based service module,face recognition module and data collection and marking module[5].

\subsection{Location Based Service Module}

Location service is based on massive location data,proceeding from the specific requirements of
The implementation steps of intelligent venue face recognition system are as follows: first,the face image data of participants is collected in advance under the face image acquisition module and will be input into the face recognition system; Second,take photos when the face image recognition system is running, and make face realtime dynamic recognition.Third,switch to the database management module,connect to the background database, and inquire the collected data information; Fourth,switch to the visual column of information data chart,and click the histogram to check the number of absence and attendance[7].The system greatly improves the efficiency of the check and the security of the exhibition,reduces the cost of manual check and security check, and is more conducive to creating a convenient,safe and reliable exhibition environment.

\subsection{Data Collection Module}

Based on the highly mature intelligent resolution monitoring system,when visitors stay in the booth,the reader will transmit the number and the label information of visitors and other data to the information management system by automatic scanning.Through storage,analysis and processing,the system can help exhibitors to find interested clients on site,provide better on-site services for clients,assist exhibitors to promote business,and improve the value of the exhibition. This not only provides more accurate and real-time data for the on-site management of relevant institutions of the exhibition, which help managers to analyze and make a decision in time,but also provides better planning data and decision-making support for the next exhibition[8]. 
computer technology and software system to convey exhibition information efficiently.This provides a platform for organizers and participants to exchange information and interact with each other.Exhibition industry should explore deeply intelligent venue solution,integrate intelligent thinking into venue information management,explore deeply and practice intelligent venue solution,explore continuously in service experience,information collection, AI system construction and other fields, and innovate business module design to meet the operational needs of intelligent conference.

\section{REFERENCES}

[1] Li,D.Y.,Zhu,C.M.RFID-based Exhibition Network Information Management System Design. Modern Computer (professional edition), 2010(13),p.87

[2] Wang,X.New Development of NUI -- Exploration of Intelligent Voice Interaction Design Mode .Scientific and Technological Innovation and Application, 2019(29), pp.35-36

[3] Anonymity.IFLYTEK AIUI3.0 Human-computer Interaction Experience [J]. Network Security and Informatization, 2018(06),p.17.

[4] Li,T.L.Research on the Development Status and Trend of Database Technology .Technology Wind, 2019(02),p.89.

[5] Hao,J.S.,Liu,R.,Xia,Y.A Tactical Cloud Storage Data Backup sSystem .New Technology and New Technique, 2019(05),p.44.

[6] Fan,X.B.,Zhang,H.J.,Zhang,X.L. Research on Interactive Visual Analysis of Enterprise Log Data .Computer Engineering and Application, 2019(01),pp.2-3.

[7] Shi,C.Design of Data Acquisition and Intelligent Data Processing System .Electronic Design Engineering,2018(16) ,pp.45-46.

[8] Zhu,Y.Y.,Zhong,L.W.Intelligent Check-in System Design of Face Recognition and Data Analysis Software Guide, 2019(06), pp.17-20.

\section{CONCLUSION}

Intelligent venue adopt modern information technology to manage all aspects of exhibition activities,relying on 